Yu-Quan Feng*, Zhi-Guo Zhong and Zi-Long Yue

\title{
Crystal structure of hexakis $\left(\mu_{2}\right.$-azido- $\left.{ }^{2} N: N\right)$ - diazido- $\kappa^{1} N$-tetrakis(phenanthroline- $\kappa^{2} N, N^{\prime}$ ) tetrazinc(II), $\mathrm{C}_{48} \mathrm{H}_{32} \mathrm{~N}_{32} \mathrm{Zn}_{4}$
}

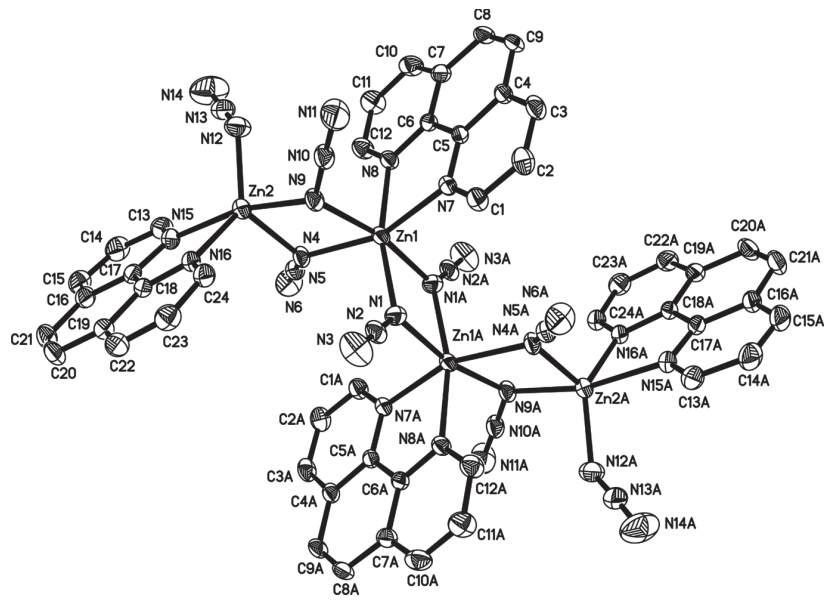

https://doi.org/10.1515/ncrs-2019-0473

Received July 6, 2019; accepted September 19, 2019; available online October 9, 2019

\section{Abstract \\ $\mathrm{C}_{48} \mathrm{H}_{32} \mathrm{~N}_{32} \mathrm{Zn}_{4}$, triclinic, $P \overline{1} \quad($ no. 2$), \quad a=9.9928(14) \AA$, $b=10.1374(14) \AA, \quad c=14.2362(19) \AA, \quad \alpha=71.710(2)^{\circ}$, $\beta=76.166(2)^{\circ}, \quad \gamma=71.086(2)^{\circ}, \quad V=1280.1(3) \AA^{3}, \quad Z=1$, $R_{\mathrm{gt}}(F)=0.0314, w R_{\text {ref }}\left(F^{2}\right)=0.0795, T=296(2) \mathrm{K}$.}

CCDC no.: 1951372

Table 1 contains crystallographic data and Table 2 contains the list of the atoms including atomic coordinates and displacement parameters.

\section{Source of material}

The mixture of $\mathrm{Zn}\left(\mathrm{NO}_{3}\right)_{2} \cdot 6 \mathrm{H}_{2} \mathrm{O}(0.20 \mathrm{~g})$, 1,10-phenanthroline $\left(\mathrm{C}_{12} \mathrm{H}_{8} \mathrm{~N}_{2}, 0.16 \mathrm{~g}\right), \mathrm{NaN}_{3}(0.10 \mathrm{~g})$ and $\mathrm{H}_{2} \mathrm{O}(3.0 \mathrm{~mL})$ was transferred into $50 \mathrm{~mL}$ Teflon-lined stainless steel vessels and heated at $160{ }^{\circ} \mathrm{C}$ for 5 days. After cooling to room temperature,

\footnotetext{
*Corresponding author: Yu-Quan Feng, College of Chemistry and Pharmacy Engineering, Nanyang Normal University, Nanyang 473061, China, e-mail: fengyuquan@nynu.edu.cn. https://orcid.org/0000-0002-3232-1680

Zhi-Guo Zhong and Zi-Long Yue: College of Chemistry and Pharmacy Engineering, Nanyang Normal University, Nanyang 473061, China
}

Table 1: Data collection and handling.

\begin{tabular}{ll}
\hline Crystal: & Yellow block \\
Size: & $0.29 \times 0.25 \times 0.20 \mathrm{~mm}$ \\
Wavelength: & Mo $K \alpha$ radiation $(0.71073 \AA)$ \\
$\mu:$ & $1.93 \mathrm{~mm}^{-1}$ \\
Diffractometer, scan mode: & Bruker APEX-II, $\varphi$ and $\omega$ \\
$\theta_{\max }$, completeness: & $26.0^{\circ}, 99 \%$ \\
$N(h k l)_{\text {measured }}, N(h k l)_{\text {unique }}, R_{\text {int }}:$ & $8227,4969,0.021$ \\
Criterion for $I_{\text {obs }}, N(h k l)_{\text {gt }}:$ & $I_{\text {obs }}>2 \sigma\left(I_{\text {obs }}\right), 3848$ \\
$N(\text { param })_{\text {refined }}:$ & 379 \\
Programs: & Bruker [1], Olex2 [2], SHELX [3] \\
\hline
\end{tabular}

the yellow block crystals were isolated, washed with distilled water and dried at room temperature.

\section{Experimental details}

$\mathrm{H}$ atoms were positioned geometrically and refined using a riding model, with $\mathrm{C}-\mathrm{H}=0.93 \AA$, with $U_{\text {iso }}(\mathrm{H})=1.2$ times $U_{\text {eq }}(\mathrm{C})$.

\section{Comment}

A large number of metal coordination compounds containing various metal cations and organic ligands have been obtained with different dimensional structures. Currently, linking metal cations and organic ligands to build coordination compounds has become a popular synthetic strategy [4]. Polydentate ligands, such as $\mathrm{N}_{3}^{-}$and $\mathrm{NCS}^{-}$, can act as either bridging or chelating ligands to construct desired metal-organic coordination compounds [4-6]. As a part of an ongoing study $[6,7]$ we have synthesized a tetra-nuclear $\mathrm{Zn}^{2+}$ coordination compound formulated $\left[\mathrm{Zn}_{4}\left(\mathrm{C}_{12} \mathrm{H}_{8} \mathrm{~N}_{2}\right)_{4}\left(\mathrm{~N}_{3}\right)_{8}\right]$ (1) containing two kinds of ligands.

Single crystal X-ray diffraction indicated that $\mathbf{1}$ belongs to the triclinic system with the space group $P \overline{1}$. The molecular structural unit of 1 contains four $\mathrm{Zn}^{2+}$ cations, four $\mathrm{C}_{12} \mathrm{H}_{8} \mathrm{~N}_{2}$ ligands and eight $\mathrm{N}_{3}{ }^{-}$anions as shown in the figure. Within the title complex $\mathrm{Zn} 1$ is six-coordinated by six $\mathrm{N}$ atoms from one $\mathrm{C}_{12} \mathrm{H}_{8} \mathrm{~N}_{2}$ ligand and four $\mathrm{N}$ atoms from four $\mathrm{N}_{3}{ }^{-}$ligands. $\mathrm{Zn} 2$ cation is five-coordinated by five $\mathrm{N}$ atoms from one $\mathrm{C}_{12} \mathrm{H}_{8} \mathrm{~N}_{2}$ ligand and three $\mathrm{N}$ atoms from three $\mathrm{N}_{3}{ }^{-}$ligands. The bond distances $\mathrm{Zn}-\mathrm{N}, \mathrm{N}-\mathrm{N}$ and the bond angles of $\mathrm{N}-\mathrm{Zn}-$ $\mathrm{N}$ are in the ranges of 1.966(3)-2.209(2) $\AA, 1.129(3)-1.196(3) \AA$ 
Table 2: Fractional atomic coordinates and isotropic or equivalent isotropic displacement parameters $\left(\AA^{2}\right)$.

\begin{tabular}{|c|c|c|c|c|}
\hline Atom & $x$ & $y$ & $z$ & $U_{\text {iso }}{ }^{*} / U_{\text {eq }}$ \\
\hline Zn1 & $0.96883(3)$ & $0.48546(3)$ & $0.39409(2)$ & $0.03393(10)$ \\
\hline $\mathrm{Zn} 2$ & $0.88633(3)$ & $0.73656(3)$ & $0.19268(2)$ & $0.03761(11)$ \\
\hline N1 & $1.1150(2)$ & $0.5437(2)$ & $0.45028(17)$ & $0.0394(5)$ \\
\hline N2 & $1.2159(3)$ & $0.5871(2)$ & $0.40522(19)$ & $0.0414(6)$ \\
\hline N3 & $1.3122(3)$ & $0.6298(3)$ & $0.3629(3)$ & $0.0736(9)$ \\
\hline N4 & $0.8220(2)$ & $0.6963(2)$ & $0.34560(17)$ & $0.0373(5)$ \\
\hline N5 & $0.7053(3)$ & $0.7486(2)$ & $0.38436(18)$ & $0.0402(6)$ \\
\hline N6 & $0.5956(3)$ & $0.7995(3)$ & $0.4215(2)$ & $0.0673(8)$ \\
\hline N7 & $1.1003(2)$ & $0.2713(2)$ & $0.39573(16)$ & $0.0330(5)$ \\
\hline N8 & $0.8249(2)$ & $0.3800(2)$ & $0.37051(17)$ & $0.0347(5)$ \\
\hline N9 & $1.0572(2)$ & $0.5552(2)$ & $0.23774(18)$ & $0.0398(6)$ \\
\hline $\mathrm{N} 10$ & $1.1254(3)$ & $0.4710(3)$ & $0.18963(19)$ & $0.0458(6)$ \\
\hline N11 & $1.1915(4)$ & $0.3893(3)$ & $0.1455(2)$ & $0.0845(11)$ \\
\hline N12 & $0.8074(3)$ & $0.6455(3)$ & $0.1233(2)$ & $0.0552(7)$ \\
\hline N13 & $0.6897(3)$ & $0.6693(3)$ & $0.1126(2)$ & $0.0553(7)$ \\
\hline N14 & $0.5763(4)$ & $0.6887(5)$ & $0.0986(3)$ & $0.1084(14)$ \\
\hline N15 & $0.7298(2)$ & $0.9507(2)$ & $0.16269(17)$ & $0.0398(6)$ \\
\hline N16 & $1.0149(2)$ & $0.8784(2)$ & $0.14230(16)$ & $0.0355(5)$ \\
\hline C1 & $1.2365(3)$ & $0.2204(3)$ & $0.4061(2)$ & $0.0421(7)$ \\
\hline $\mathrm{H} 1$ & $1.2821(3)$ & $0.2812(3)$ & $0.4151(2)$ & $0.0505(8)^{\star}$ \\
\hline $\mathrm{C} 2$ & $1.3152(3)$ & $0.0802(3)$ & $0.4041(2)$ & $0.0489(8)$ \\
\hline $\mathrm{H} 2$ & $1.4111(3)$ & $0.0483(3)$ & $0.4121(2)$ & $0.0587(9)^{\star}$ \\
\hline $\mathrm{C} 3$ & $1.2506(3)$ & $-0.0093(3)$ & $0.3904(2)$ & $0.0464(7)$ \\
\hline $\mathrm{H} 3$ & $1.3021(3)$ & $-0.1033(3)$ & $0.3886(2)$ & $0.0557(9)^{\star}$ \\
\hline $\mathrm{C} 4$ & $1.1062(3)$ & $0.0401(3)$ & $0.37888(19)$ & $0.0368(6)$ \\
\hline $\mathrm{C} 5$ & $1.0357(3)$ & $0.1840(3)$ & $0.38149(18)$ & $0.0310(6)$ \\
\hline $\mathrm{C} 6$ & $0.8880(3)$ & $0.2409(3)$ & $0.36952(19)$ & $0.0316(6)$ \\
\hline $\mathrm{C} 7$ & $0.8151(3)$ & $0.1526(3)$ & $0.3572(2)$ & $0.0391(6)$ \\
\hline $\mathrm{C} 8$ & $0.8895(3)$ & $0.0064(3)$ & $0.3575(2)$ & $0.0452(7)$ \\
\hline $\mathrm{H} 8$ & $0.8406(3)$ & $-0.0535(3)$ & $0.3511(2)$ & $0.0543(9)^{*}$ \\
\hline $\mathrm{C} 9$ & $1.0275(3)$ & $-0.0461(3)$ & $0.3669(2)$ & $0.0450(7)$ \\
\hline $\mathrm{H} 9$ & $1.0735(3)$ & $-0.1414(3)$ & $0.3654(2)$ & $0.0539(9)^{\star}$ \\
\hline C10 & $0.6716(3)$ & $0.2133(3)$ & $0.3461(2)$ & $0.0512(8)$ \\
\hline $\mathrm{H} 10$ & $0.6186(3)$ & $0.1577(3)$ & $0.3388(2)$ & $0.0615(9)^{\star}$ \\
\hline C11 & $0.6094(3)$ & $0.3530(4)$ & $0.3460(3)$ & $0.0557(8)$ \\
\hline H11 & $0.5140(3)$ & $0.3948(4)$ & $0.3373(3)$ & $0.0668(10)^{\star}$ \\
\hline C12 & $0.6888(3)$ & $0.4329(3)$ & $0.3588(2)$ & $0.0470(7)$ \\
\hline $\mathrm{H} 12$ & $0.6445(3)$ & $0.5288(3)$ & $0.3593(2)$ & $0.0564(9)^{\star}$ \\
\hline C13 & $0.5884(3)$ & $0.9844(3)$ & $0.1731(2)$ & $0.0498(8)$ \\
\hline H13 & $0.5437(3)$ & $0.9103(3)$ & $0.1969(2)$ & $0.0597(9)^{\star}$ \\
\hline C14 & $0.5045(3)$ & $1.1257(4)$ & $0.1500(3)$ & $0.0586(9)$ \\
\hline H14 & $0.4055(3)$ & $1.1450(4)$ & $0.1576(3)$ & $0.0703(11)^{\star}$ \\
\hline C15 & $0.5667(4)$ & $1.2352(4)$ & $0.1163(2)$ & $0.0561(9)$ \\
\hline H15 & $0.5111(4)$ & $1.3303(4)$ & $0.1003(2)$ & $0.0674(10)^{\star}$ \\
\hline C16 & $0.7156(3)$ & $1.2047(3)$ & $0.1058(2)$ & $0.0459(7)$ \\
\hline C17 & $0.7924(3)$ & $1.0595(3)$ & $0.1297(2)$ & $0.0375(6)$ \\
\hline C18 & $0.9458(3)$ & $1.0205(3)$ & $0.12060(19)$ & $0.0359(6)$ \\
\hline C19 & $1.0153(3)$ & $1.1295(3)$ & $0.0917(2)$ & $0.0429(7)$ \\
\hline $\mathrm{C} 20$ & $0.9337(4)$ & $1.2765(3)$ & $0.0690(2)$ & $0.0540(8)$ \\
\hline $\mathrm{H} 20$ & $0.9801(4)$ & $1.3491(3)$ & $0.0497(2)$ & $0.0648(10)^{\star}$ \\
\hline $\mathrm{C} 21$ & $0.7913(4)$ & $1.3119(3)$ & $0.0750(2)$ & $0.0579(9)$ \\
\hline $\mathrm{H} 21$ & $0.7407(4)$ & $1.4088(3)$ & $0.0588(2)$ & $0.0695(11)^{\star}$ \\
\hline
\end{tabular}

Table 2 (continued)

\begin{tabular}{lrrrr}
\hline Atom & $\boldsymbol{x}$ & $\boldsymbol{y}$ & $\boldsymbol{z}$ & $\boldsymbol{U}_{\text {iso }}{ }^{*} \boldsymbol{U}_{\text {eq }}$ \\
\hline $\mathrm{C} 22$ & $1.1637(3)$ & $1.0852(3)$ & $0.0869(2)$ & $0.0512(8)$ \\
$\mathrm{H} 22$ & $1.2150(3)$ & $1.1536(3)$ & $0.0687(2)$ & $0.0614(9)^{*}$ \\
$\mathrm{C} 23$ & $1.2336(3)$ & $0.9424(4)$ & $0.1088(2)$ & $0.0511(8)$ \\
$\mathrm{H} 23$ & $1.3326(3)$ & $0.9124(4)$ & $0.1049(2)$ & $0.0614(9)^{\star}$ \\
$\mathrm{C} 24$ & $1.1549(3)$ & $0.8419(3)$ & $0.1369(2)$ & $0.0420(7)$ \\
$\mathrm{H} 24$ & $1.2034(3)$ & $0.7443(3)$ & $0.1527(2)$ & $0.0504(8)^{*}$ \\
\hline
\end{tabular}

and $76.53(8)-168.68(9)^{\circ}$, respectively. These above mentioned bond distances and angles are all in their normal ranges. They can be compared with those previously reported compounds [8]. By means of such connected modes, a tetra-nuclear coordination compound was generated. The distances of $\mathrm{Zn} \cdots \mathrm{Zn}$ are $3.251 \AA$ and $3.338 \AA$. It is worth noting that the eight $\mathrm{N}_{3}{ }^{-}$ligands exhibited two types of coordination fashions: six $\mathrm{N}_{3}{ }^{-}$ligands as bridged ligands linked to two $\mathrm{Zn}^{2+}$ cations (Zn1 and $\mathrm{Zn} 2$ ) and two $\mathrm{N}_{3}{ }^{-}$ligands as terminal ligands connected to $\mathrm{Zn} 2$. In addition, $\mathrm{C}-\mathrm{H} \cdots \mathrm{N}$ hydrogen bonds are found in the packing structure of $\mathbf{1}$.

Acknowledgements: This work was financially supported by the National Natural Science Foundation of China (no. 21601095).

\section{References}

1. Bruker. APEX2, SAINT v7.60A and SAINT v7.60A. Bruker AXS Inc., Madison, WI, USA (2008).

2. Dolomanov, O. V.; Bourhis, L. J.; Gildea, R. J.; Howard, J. A. K.; Puschmann, H.: OLEX2: a complete structure solution, refinement and analysis program. J. Appl. Crystallogr 42 (2009) 339-341.

3. Sheldrick, G. M.: Crystal structure refinement with SHELXL. Acta Crystallogr. C71 (2015) 3-8.

4. Gao, E.-Q.; Bai, S.-Q.; Wang, C.-F.; Yue, Y.-F.; Yan, C.-H.: Structural and magnetic properties of three one-dimensional azidobridged copper(II) and manganese(II) coordination polymers. Inorg. Chem. 42 (2003) 8456-8464.

5. Yue, Y.-F.; Gao, E.-Q.; Bai, S.-Q.; He, Z.; Yan, C.-H.: Highdimensional azido-bridged cadmium(II) polymeric complexes with bis(bidentate) diazine ligands. CrystEngComm 6 (2004) 549-555.

6. Zhang, W.; Feng, Y.-Q.: A novel dinuclear bismuth(III) coordination compound: bis( $\mu$-pyridine-2,6-dicarboxylato)- $-\kappa^{4} O^{2}, N$, $O^{6}: O^{6} ; \mathrm{K}^{4} O^{2}: O^{21}, N, O^{6}$-bis[(azido- $\left.\mathrm{K} N\right)(1,10$-phenanthroline$\left.\mathrm{K}^{2} N, N^{\prime}\right)$ bismuth(III)]tetrahydrate. Acta Crystallogr. C70 (2014) 562-565.

7. Feng, X.; Feng, Y.-Q.; Liu, L.; Wang, L.-Y.; Song, H.-L.; Ng, S.-W.: A series of $\mathrm{Zn}-4 \mathrm{f}$ heterometallic coordination polymers and a zinc complex containing a flexible mixed donor dicarboxylate ligand. Dalton Trans. 42 (2013) 7741-7754.

8. Sun, J.-Y.; Wang, X.-X.: Di- $\mu$-1,1-azido-bis[azido(5,5'-dimethyl2,2'-bipyridine- $\left.{ }^{2} N, N^{\prime}\right)$ zinc(II)]. Acta Crystallogr. E63 (2007) m1138-m1139. 